\title{
Architecture and Performance of A MAC Protocol for The HFC System
}

\author{
Shengming Jiang $\dagger$, Danny H.K. Tsang $\dagger$, Samuel T. Chanson $\dagger^{\dagger}$ \\ Hong Kong University of Science \& Technology \\ Clear Water Bay, Kowloon, Hong Kong \\ $\dagger\{$ eejiang, eetsang $\} @$ ee.ust.hk, $\dagger \dagger$ chanson@cs.ust.hk
}

\begin{abstract}
The IEEE 802.14 standard committee is currently working on a project to find a costeffective means of providing access to integrated networks for people to enjoy multimedia programs and to work at home. An advanced system based on the CATV system called Hybrid Fiber Coax (HFC) is being studied. Since some properties of the HFC system preclude the possibility of directly using existing medium access control (MAC) protocols for its data link layer, a MAC scheme based on time division is discussed in this paper. This MAC scheme can be extended for wireless networks.
\end{abstract}

Key words: MAC, HFC, ATM, Multimedia, SCCA/TD

\section{Introduction}

As ATM technology progresses, researchers are looking for a cost-effective means of providing home access to integrated networks so that people can enjoy multimedia programs and work at home. Due to the large geographical coverage of existing CATV systems, an advanced system based on CATV called Hybrid Fiber Coax (HFC) is being studied by the IEEE 802.14 Project Committee [1]. This system will likely use ATM technology to provide multiple services to home such as telephone, TV and information retrieval. As shown in Figure 1, a simple HFC system may be abstracted into a dualbranch-bus based network consisting of a distributing hub (called headend in this paper) and the fiber nodes (or simply nodes). The coaxial plant which connects residential zones is extended from the fiber nodes. The two buses can be looped at the headend to form a single bus. Several simple HFC systems can be connected via their distributing hubs. 


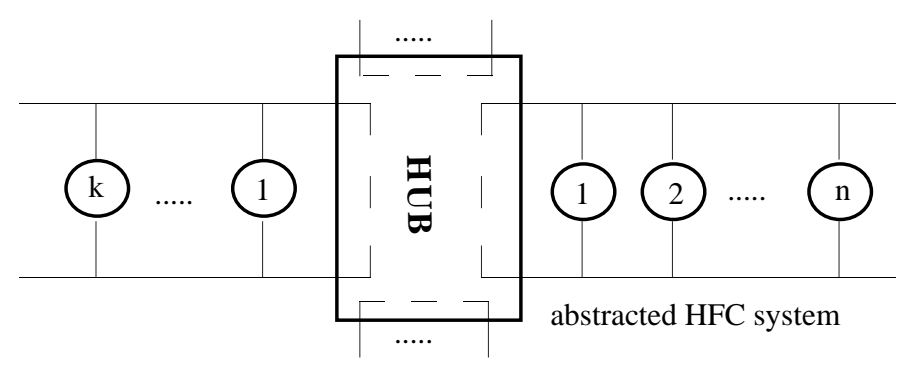

Figure 1 Topology of the HFC system

In the HFC system, the downstream band and upstream band operate asymmetrically at different frequencies, and a node has only one link to each bus. In the downstream direction (from the headend to the nodes) broadcast mode is used, and the headend is the only transmitter. This makes control of the downstream band simple. In the upstream direction (from the nodes to the headend), the nodes share the upstream band for transmission, and contention access mode is used. To allow the nodes to share the upstream band in a fair manner ${ }^{1}$, an efficient medium access control (MAC) protocol is necessary. This protocol has to be cost-effective and efficient for long distance communication, supporting a wide range of transmission rates as well as both analog and digital broadband services [2]. Unfortunately, these properties and requirements make it difficult to use existing MAC protocols directly for the following reasons:

1) The ring-based protocols such as Token-Ring [5], FDDI [5], ATMR [3] and Orwell [4] are not suitable for the dual-branch-bus topology required by the HFC system. On the other hand, both CSMA/CD and Token-Bus [5] are suitable for the HFC topology. However, CSMA/CD cannot guarantee bounded delay and is only suitable for relatively short distances. Since different frequencies are used for transmitting and receiving in the HFC system, messages for inter-node communication need to be retransmitted by the headend. Retransmission from the headend will result in long token-passing time in tokenpassing-based schemes like Token-Bus, especially when the bus is very long and covers a wide area. Another weakness of Token-Bus is that it requires the users to remain on-line to be included in the logical ring which needs to be reconfigured whenever users are added or removed from the network.

\footnotetext{
${ }^{1}$ By 'a fair manner', we mean each node will have equal opportunity of accessing the bus.
} 
2) It is difficult to use MAC protocols based on the slotted media approach like DQDB [5] and CRMA [6]. These protocols often require a node to have two links to each bus for data insertion. Moreover, DQDB needs two headends and CRMA requires a folded bus while there is only one headend and the topology is required to be dual-branch-bus in the HFC system. Although the two buses in the HFC system can be looped to form a folded bus like CRMA, the outbound bus (from the headend to the nodes) is reserved for transmission, and the inbound bus (from the nodes to the headend) for receiving in the folded-bus CRMA. This is opposite to the HFC system where the downstream band is for receiving and upstream band for transmission. In addition, to divide bandwidth into slots, the slot header has to be broadcast to the nodes continuously. In the HFC system, this can be done by the headend and the slot header will be broadcast to the nodes via the downstream band. However, in this case, the downstream band cannot be used by the nodes for transmission.

With regards to the special position of the headend in the HFC system, a MAC scheme based on centralized control is a natural choice. One such scheme is the traditional polling system such as Roll-call Polling [9]. However, these systems have been designed for low or medium speed data applications under the assumption that the propagation delay is negligible compared to the transmission time. This assumption is not true in high speed networks [7]. In this paper, we will discuss a new MAC scheme called Self-Control Cycle Access with Time Division (SCCA/TD). The philosophy of SCCA/TD is similar to that of Time Division Multiplex Access (TDMA) and Packet Reservation Multiple Access (PRMA [8]) in terms of time division and cyclic control for bandwidth allocation. The main differences are as follows:

1) In TDMA and PRMA, the users have to contend for idle time slots for bandwidth reservation at the beginning of transmission. This competition is based on users monitoring the broadcast messages on uplink channel usage in the downlink channel. This scheme is unfair since the physical positions of the users are fixed and the broadcast messages come from the headend to the nodes in the HFC system. As a consequence, the nodes closer to the headend will have more opportunities for successful reservation than the ones further from the headend. Therefore, in SCCA/TD, a small amount of bandwidth is permanently reserved for each node to make requests or transmission, which provides quick access to the medium. 
2) In TDMA and PRMA, bandwidth is slotted and the users with successful reservation have to synchronize the downlink channel for using the reserved time slots. This requires the control center (e.g., base station or headend) to signal the users repeatedly; and any signal error will degrade network performance. In SCCA/TD, bandwidth is unslotted, and scheduling at the headend prevents different nodes from using the same time slot. After receiving a positive acknowledgment from the headend, the nodes can continue to use the same allocation periodically in subsequent cycles, without any additional control from the headend. Therefore, SCCA/TD can simultaneously support any types of MAC protocol data units and different transmission rates.

The rest of the paper is organized as follows. The principle of SCCA/TD is introduced in Section 2. In Section 3, a prototype implementation of SCCA/TD is described, and simulation studies on this prototype are give in Section 4. Finally Section 5 concludes the paper.

\section{Introduction to SCCA/TD}

SCCA/TD is a reservation based MAC scheme which allows the nodes to share the upstream bandwidth. A node normally needs to make requests for bandwidth through the upstream band. The headend will respond to the requesting nodes through the downstream band. The upstream bandwidth is divided into equal time segments called 'cycles'. A cycle is divided again into fractions which are assigned to the nodes according to their requests. As shown in Figure 2, the same epoch of the cycle is repeated periodically as long as the requests of the nodes are unchanged. That is, the quota and position of the bandwidth allocation to a given node in each cycle are not changed. In this way, a node can access the medium simply knowing the relative position of its assignment in the cycle. The granted bandwidth can be used by the nodes for transmitting data or submitting requests.

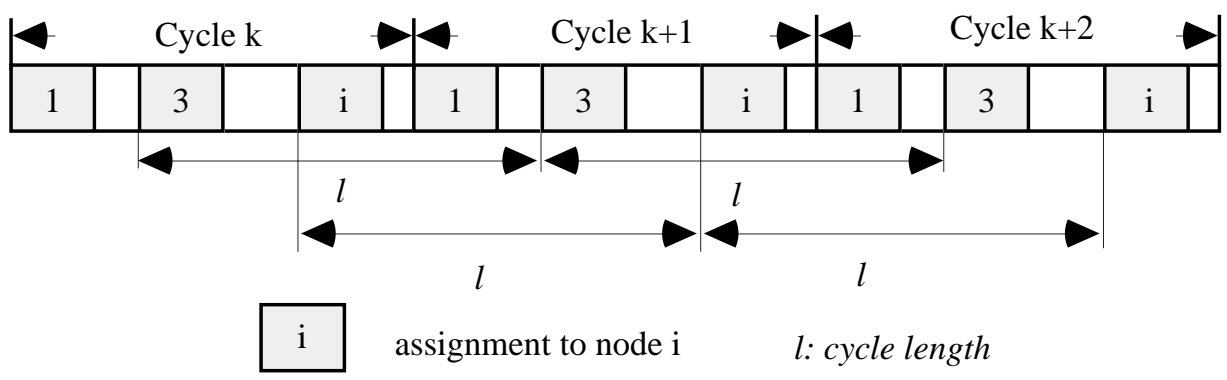

Figure 2 The principle of SCCA/TD 
As mentioned earlier, the upstream band and downstream band use different frequencies so there is no signal interference between broadcasting and receiving messages in the same bus. Therefore, SCCA/TD can run independently in each bus. However, to simplify the description and simulation, in this paper we will assume only one bus is used as the upstream band for transmission by the nodes (called upstream band bus) and the other as the downstream band for broadcasting from the headend (called downstream band bus), as shown in Figure 4.

\subsection{Cycle Structure}

A cycle is divided into the static reservation segment (SRS) and the dynamic reservation segment (DRS) as shown in Figure 3. SRS gives each node a basic bandwidth assignment periodically in each cycle. A node can use the SRS assignment to submit requests or transmit small amounts of data. DRS, on the other hand, provides bandwidth dynamically to the nodes according to their requests. The advantage of using SRS is that it provides the nodes quick access to the medium. However, if a node has nothing to transmit, its assignment will be wasted. Requiring several nodes to share the same assignment alternately can improve efficiency. When a node has a large amount of data to transmit, it needs to get the requested bandwidth from DRS. To this end, the node submits a request to the headend carried by a special packet containing the node's identifier, transmission speed, request type and other related parameters. The headend will respond with a special confirmation packet.

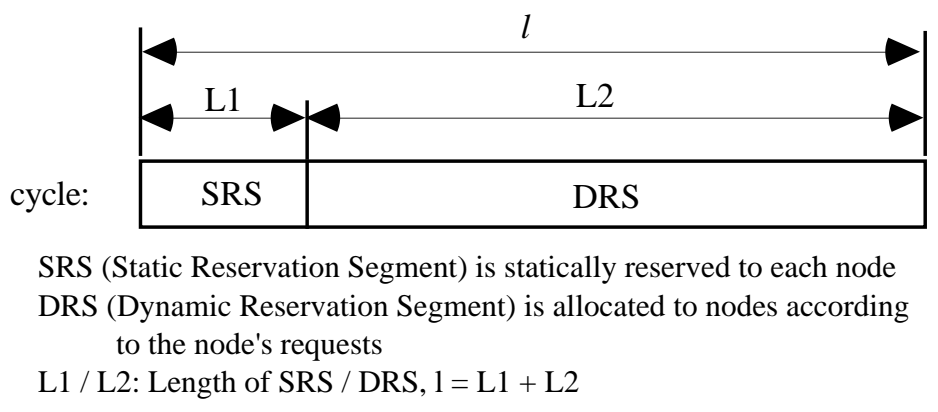

Figure 3 The cycle structure

There are two types of requests that a node can submit to the headend: Gross Bandwidth Request (GBR) and Cyclic Bandwidth Request (CBR). With GBR, a node informs the headend of the total amount of bandwidth it needs, and the headend serves the request in consecutive cycles if the request cannot be satisfied within a single cycle. In this 
case, the requesting node will get the total required bandwidth in multiple cycles, each providing only a fraction of the total required bandwidth. With CBR, a node informs the headend of the bandwidth it requires in each cycle. If the request is granted, the node gets the same quota of bandwidth in the same relative location in each subsequent cycle until the end of transmission. When the node finishes transmission, it has to send a message to the headend to relinquish its DRS reservation. The first type of request is suitable for bursty traffic such as data while the second type is suitable for periodic traffic such as voice and video.

For both SRS and DRS, the nodes can adopt the self-control cyclic access mechanism to use the bandwidth after receiving confirmation without additional control from the headend. That is, the headend notifies the requesting node the starting time of its bandwidth assignment, the quota of bandwidth that the node can use in each cycle and the cycle length, i.e., the period in which the node can use its assignment until either the expiration of its grant (for GBR request) or the end of transmission (for CBR request). This scheme can be implemented with timers or counters for fixed length MAC protocol data units.

A more complex scheme with hierarchical cycle structure can be developed based on SCCA/TD, which can satisfy different delay requirements of the applications simultaneously. This can be done by dividing the main cycle shown in Figure 3 into several sub-cycles, each with the same length equal to $l / \Omega$, where $\Omega$ is the number of sub-cycles in the main cycle $(l / \Omega>\mathrm{L} 1)$. For example, in a node whose application requires the access delay to be smaller than $l$, the headend can allocate two assignments to it in each main cycle, and the access delay is $l / 2(\Omega=2)$. For other nodes whose applications are not sensitive to delay, the headend can allocate a quota to them in each main cycle or even every two main cycles. However, in this paper only the one cycle case will be discussed.

\subsection{Scheduling Schemes}

Collisions burden networks with extra work for collision detection and resolution, and wastes bandwidth. This is especially undesirable in high-speed networks. Therefore, SCCA/TD is designed to be collision-free. The scheduling done by the headend is the key to collision avoidance. The bandwidth allocation mechanism has to maintain fairness for the nodes to share bandwidth while avoiding collision to improve network utilization. There are many alternatives for the allocation policy. A simple scheme would be for the headend 
to collect the requests and serve them one by one in round robin. This scheme is used in the simulation studies below. In the following, we discuss two collision avoidance approaches. The following parameters are used in the discussion:

$n$ - total number of nodes in the network (see Figure 4);

$m$ - total number of nodes simultaneously sharing the same cycle;

$l$ - cycle length;

$d(\mathrm{i}, \mathrm{j})$ - distance between nodes $\mathrm{i}$ and $\mathrm{j}$;

$d$ - distance between nodes 1 and n, i.e., maximum propagation delay;

$\mu$ - bandwidth utilization measured in terms of the total time used for transmission within a cycle over the cycle length.

(for simplicity, $l, d(\mathrm{i}, \mathrm{j})$ and $d$ are all measured in time units)

\section{a) The Efficient Approach (EA)}

For this approach, we suppose that the signal propagation in the upstream band bus is unidirectional towards the headend, and the sequence of bandwidth assignment to the nodes in each cycle is in accordance with the sequence of their physical positions in the network. In this case, the cycle always begins with the assignment to the node closest to the headend. The propagation delay between adjacent nodes is taken into account to maximize bandwidth utilization which can go up to 1 . This can be explained by Figure 4, where the cycle is fully shared by $m$ active nodes.

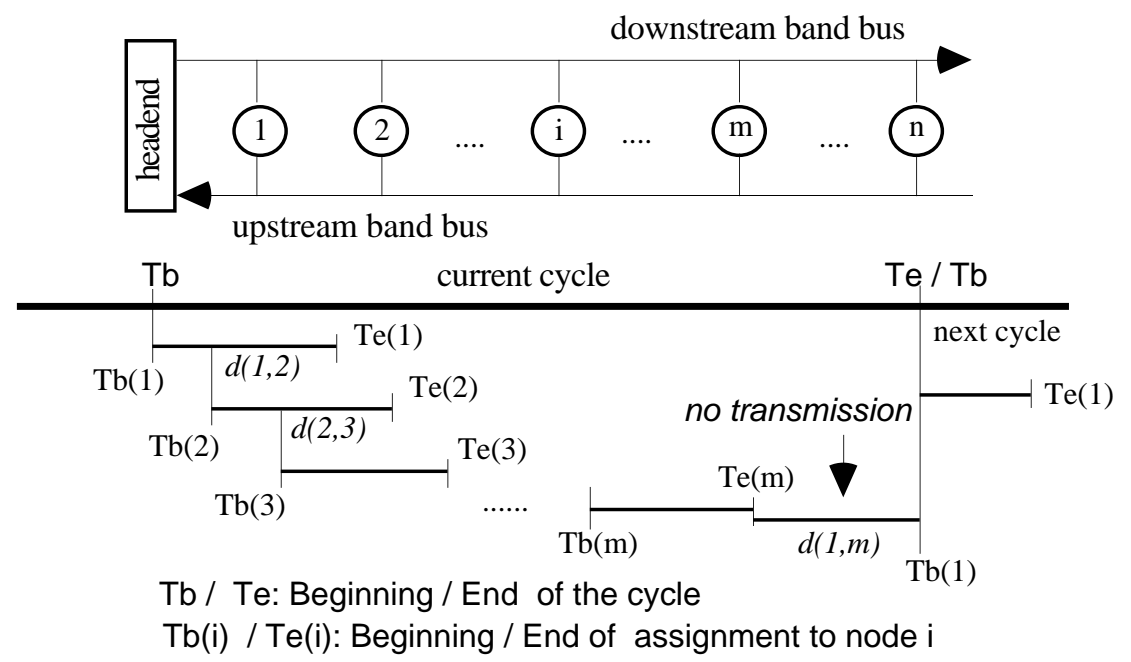

Figure 4 Diagram of cycle division with EA 
Notice that an active node $i+1$ can advance its transmission by $d(i, i+1)$ ahead of the end of transmission of node $i$ due to propagation delay. The total time advanced within a cycle is equal to $\sum d(\mathrm{i}, \mathrm{i}+1)=d(1, \mathrm{~m}) \quad(\mathrm{i}=1,2, . . m-1)$. When the last active node $m$ finishes transmission, time $d(1, \mathrm{~m})$ is required to allow its traffic pass through all the downstream nodes before the beginning of the next cycle. This time is compensated by the total time advanced by all the nodes. Therefore, EA can give high bandwidth utilization independent of network size and cycle length. However, if no bandwidth in a suitable position in the cycle is available to a new requesting node, the headend has to shift the positions of some existing assignments in order to accept new requests. For example, as shown in Figure 5, if nodes 3, 4 and 8 become active, there is no free segment suitable to them even though there are several free segments in the cycle. By shifting the bandwidth assignments for nodes 1, 2 and 7 to the left (occupying some of the unassigned space), some spaces are made available for the new requesting nodes in the right position. In this case, the headend needs to send messages to the affected nodes informing them their new bandwidth assignments in the cycle. Shifting bandwidth assignments can provide more flexible and efficient bandwidth allocation at the expense of complexity.

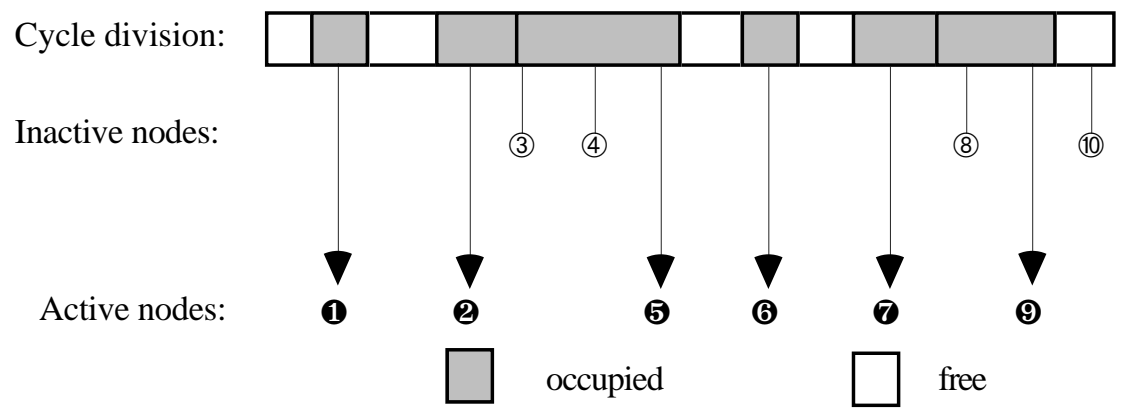

Figure 5 Assignment in a cycle versus the physical position of the nodes

\section{b) The Simple Approach (SA)}

With this approach, the relative physical positions of the nodes in the network are not taken into account. The maximum distance (i.e., $d$ ) is used as the distance between any pair of nodes. In this case, when an active node finishes its assignment in a cycle, the next active node has to wait $d$ time units before starting its transmission. $\mu=1-m * \Delta$, where $\Delta=d$ / $l$, is the bandwidth wastage per active node due to the gap placed between adjacent assignments for avoiding collision. As shown in Figure 6, bandwidth utilization is inversely proportional to the number of active nodes for a given cycle length and network size. The maximum bandwidth utilization is bounded by $1-\Delta$. Therefore, this approach is 
suitable for small size networks only since it is easy to get small $\Delta \mathrm{s}$ with long cycles in this case.

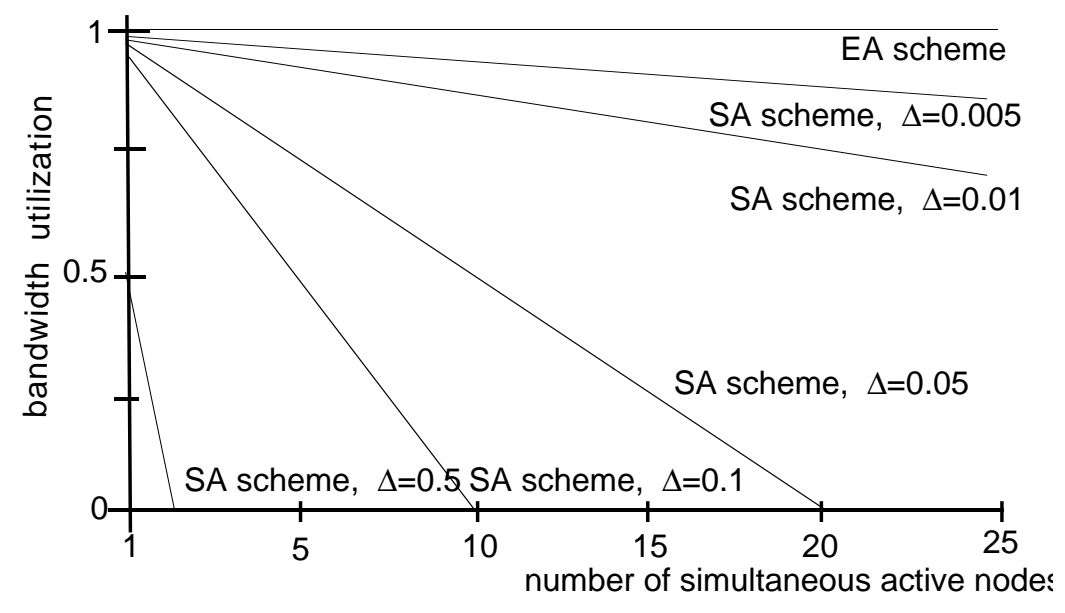

Figure 6 Bandwidth utilization in SA and EA schemes

SCCA/TD with SA can be extended for use in wireless networks since SA does not take into account the relative physical positions of the nodes for bandwidth allocation. This permits the nodes to move within a given geographic area, which is the case in wireless environments. The primary modification necessary to adapt SCCA/TD with SA for wireless networks is the access mode to the SRS segment since it uses static reservation which is not suitable for mobile users. Random access modes like ALOHA can be used to control the access to this segment. In this case, the base station (BS) in wireless networks plays the role as the headend in the HFC system. When hand-off occurs for a given mobile, the current serving BS informs the neighboring BSs its request and the remaining allocation quota. The mobile will receive the starting time of transmission, the cycle length and the frequency from the new serving BS to set the controller for transmission. Detailed discussion on this issue is beyond the scope of this paper.

\section{A Prototype Implementations of SCCA/TD}

In this section, we present a prototype implementation of SCCA/CD by describing the functional structure of the main components in the SCCA/CD scheme. The ATM cell is used as the MAC protocol data unit.

\subsection{Overview of System Structure}




\section{i) MAC Cell}

The MAC cell is used to carry the message of the MAC layer such as requests from nodes, acknowledgment from the headend, the cycle length and other information related to MAC layer control. This cell has the same format as the ATM cell, and can be identified by a special GFC (Generic Flow Control) setting. The MAC cell format is given in Figure 7. However, the length of each field needs further studies, and some new fields may be necessary for other functions.

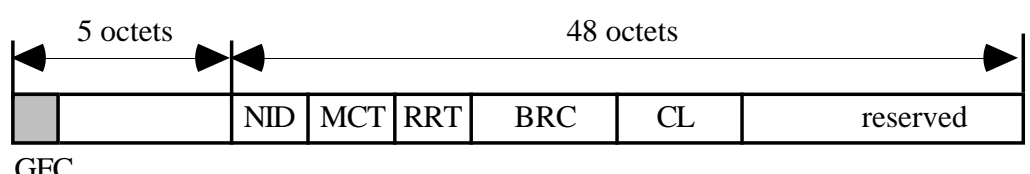

Figure 7 MAC cell format

NID (Node Identifier, 12 bits): the identifier must be unique in the given HFC system .

MCT (MAC Cell Type, 4 bits): there are two types of MAC cells - one from the nodes to the headend (called upstream MAC cell: UMC) and the other from the headend to the nodes (called downstream MAC cell: DMC).

$\underline{\text { RRT }}$ (Request / Response Type, 4 bits, used with MCT ): if MCT = UMC, it contains the request type (GBR or CBR, see Section 2.1); if $\mathrm{MCT}=\mathrm{DMC}$, it is used to carry the headend's acknowledgment (positive or negative).

BRC (Bandwidth Requested / Confirmed, 2 octets, used with MCT and RRT): when MCT $=\mathrm{UMC}$, it indicates the amount of bandwidth required by node NID. Similarly, if $\mathrm{MCT}=\mathrm{DMC}$, it indicates the amount of bandwidth confirmed or refused by the headend.

$\underline{\mathrm{CL}}$ (Cycle Length, 2 octets): the period with which a node can repeat using the assignment.

ii) Node

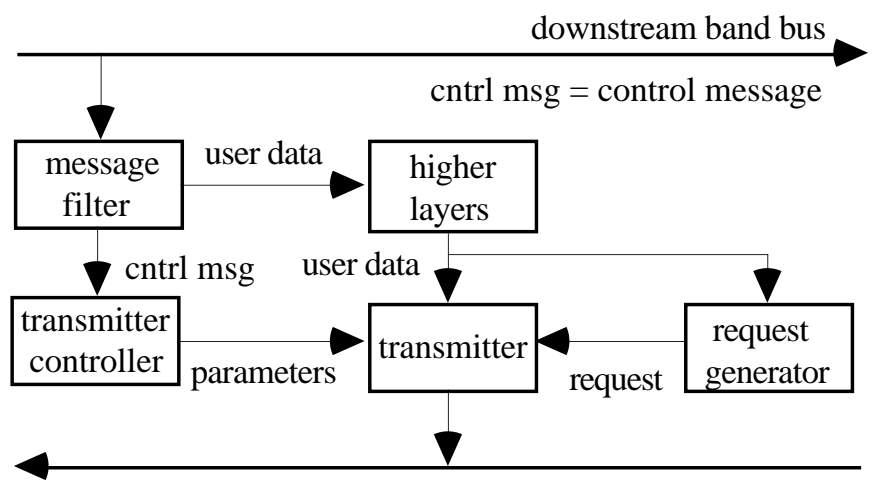

upstream band bus 
Figure 8 Functional structure of a node

As shown in Figure 8, the request generator submits a request to the headend by sending a MAC cell when the number of the data cells accumulated in the MAC layer exceeds the request threshold (i.e., T, refer to Section 3.2 for details). The MAC cells have a higher priority than the data cells and are sent to the upstream band bus by the transmitter before any data cells. A node copies all the messages from the downstream band bus and selects the ones destined to it using the message filter. The MAC cell is passed to the transmitter controller to determine the parameters used to set the transmitter.

iii) Headend

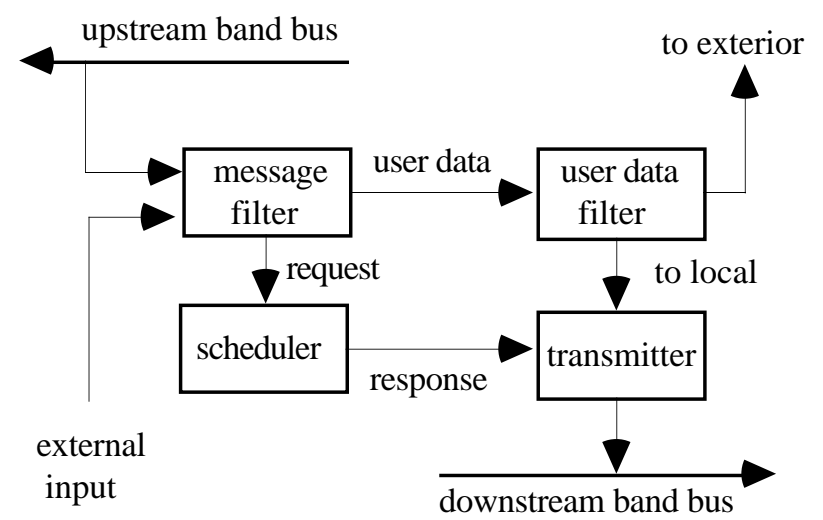

Figure 9 Functional structure of the headend

All the messages arriving at the headend are filtered according to the request message and user data. The requests are passed to the scheduler for processing. The headend will send the response to the requesting nodes via the transmitter using the downstream band bus. The user data are examined to see if they are local or external. The former is transferred to the downstream band bus and the later to the external link.

\subsection{Implementation of Scheduling Schemes}

We have described two scheduling approaches in Section 2.2. In this section, we describe possible ways to implement these two scheduling schemes. The parameters used are defined below:

$\mathrm{M}=$ maximum quota per cycle for each node; 
$\mathrm{q}=$ bandwidth quota allocated to a node per cycle;

$\mathrm{R}=$ bandwidth requested by a node to be honored;

$\mathrm{T}=$ request threshold. A node can make a request only when the number of "nonrequested" cells accumulated in the buffer exceeds this threshold. The "non-requested" cells are ones for which the node has not made a request.

$\mathrm{F}=$ the largest free segment in the cycle;

$\mathrm{I}=$ minimum gap between two successive assignments in the cycle to avoid collision; (refer to Section 2.2, Figures 3 and 4 for the definitions of other parameters)

\subsubsection{SA Implementation}

The implementation of this approach consists of the following steps:

1) Find $F$ in the cycle by scanning the entire cycle. $\mathrm{Tb}$ and $\mathrm{Te}$ of $\mathrm{F}$ are denoted by Tb_max and Te_max;

2) If $\mathrm{F} \leq \mathrm{I}$, then stop the allocation process;

3) Calculate $\mathrm{q}, \mathrm{Tb}$ and $\mathrm{Te}$ for the request to be honored:

$$
\begin{aligned}
& \mathrm{q}=\min (\mathrm{M}, \mathrm{F}-\mathrm{I}, \mathrm{R}) ; \\
& \mathrm{Tb}=\mathrm{Tb} \_\max ; \\
& \mathrm{Te}=\mathrm{Tb}+\mathrm{q}+\mathrm{I} ;
\end{aligned}
$$

4) Calculate the starting time for the node's transmission. The queuing delay experienced by the MAC cell at the headend and the propagation delay between the headend and the node under consideration (the default value is $d$ for scheme SA) are two major components in computing the starting time.

As mentioned in Section 2.2, the bandwidth utilization $(\mu)$ of this scheme depends on the number of nodes $(m)$ simultaneously sharing the cycle. The average bandwidth utilization $\mu$ is equal to $(1-\mathrm{A} * \Delta)$, where $\mathrm{A}=\mathrm{L} 2 /(\mathrm{Q}+\mathrm{I})$ is the average number of simultaneous active nodes and $\mathrm{Q}$ is the average bandwidth quota allocated to a node. When the system is saturated (i.e., the nodes always have traffic to transmit), $\mathrm{Q} \approx \mathrm{M}$. Hence a larger M results in a higher $\mu$. The largest M is equal to (L2 - I) which leads to the maximum $\mu=1-\Delta$ as specified in Section 2.2. In this case, longer cycles help to increase $\mu$. However, if the cycle is too long for the nodes to use up the allocation, bandwidth will be wasted. Therefore, achieving high $\mu$ with longer cycles also depends on the input traffic density of the nodes. 


\subsubsection{EA Implementation}

The implementation of this scheme normally requires the headend to re-arrange the positions of some existing assignments in the cycles in order to accept new requests. Here, we give a quasi-EA implementation (qEA) which does not need to perform the above operation but can still achieve a higher bandwidth utilization than the SA scheme. The basic idea is that when allocating an assignment in the cycle for a node, the position of the assignment in the cycle is determined by the node's physical position in the network to avoid irregular cycle divisions. This can be done using the algorithm described below.

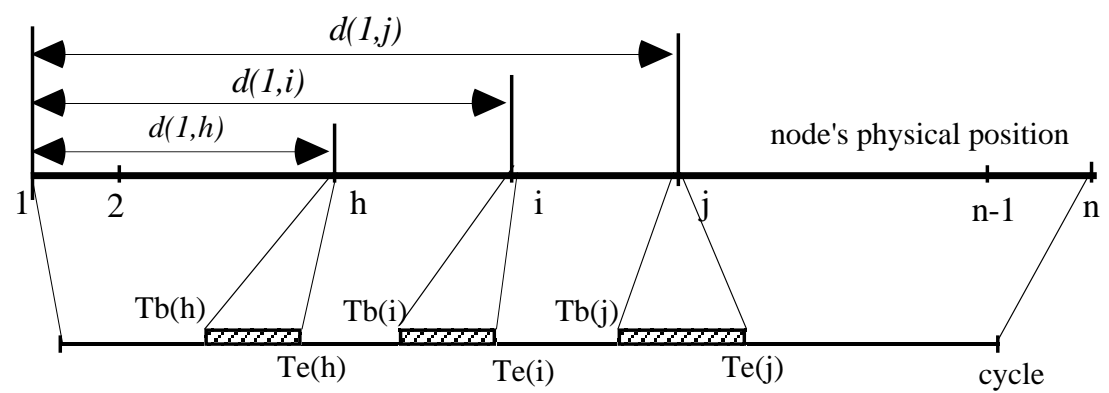

Figure 10 Node's physical position and the assignment in cycle with qEA

In Figure 10, node $i$ is the current node under consideration, nodes $h$ and $j$ are respectively the downstream and upstream active nodes which are the closest to node $i$. $d(1, \mathrm{~h}), d(1, \mathrm{i})$ and $d(1, \mathrm{j})$ are the propagation delays from node 1 . The available bandwidth between the assignments to nodes $\mathrm{h}$ and $\mathrm{j}, \mathrm{B}$, is equal to $\mathrm{Tb}(\mathrm{j})-\mathrm{Te}(\mathrm{h})+d(1, \mathrm{j})-d(1, \mathrm{~h})$, by taking into account the propagation delay between them. The algorithm to determine $\mathrm{Tb}$ and Te for node $i$ is as follows:

$$
\begin{aligned}
& \operatorname{IF} \min (\mathrm{M}, \mathrm{R}) \geq \mathrm{B} \text { THEN }\{ \\
& \mathrm{q}=\mathrm{B} \\
& \operatorname{Tb}(\mathrm{i})=\mathrm{Te}(\mathrm{h})-[d(1, \mathrm{i})-d(1, \mathrm{~h})]\}
\end{aligned}
$$

ELSE \{

$$
\begin{aligned}
& \mathrm{q}=\min (\mathrm{M}, \mathrm{R}) \\
& \operatorname{IF}(\mathrm{j}-\mathrm{h}-2)>0 \text { THEN } \\
& \quad \mathrm{S}=(\mathrm{B}-\mathrm{q}) *(\mathrm{i}-\mathrm{h}-1) /(\mathrm{j}-\mathrm{h}-2)
\end{aligned}
$$

ELSE

$$
\begin{gathered}
\mathbf{S}=0 \\
\operatorname{Tb}(\mathbf{i})=\operatorname{Te}(\mathbf{h})-[d(1, \mathbf{i})-d(1, \mathbf{h})]+\mathbf{S}\}
\end{gathered}
$$


$\operatorname{Te}(\mathrm{i})=\mathrm{Tb}(\mathrm{i})+\mathrm{q}$

If the allowable quota for node $\mathrm{i}, \min (\mathrm{M}, \mathrm{R})$, is larger than $\mathrm{B}$, then the assignment to node $\mathrm{i}$ is set to $\mathrm{B}$, and the assignment is made to follow right after the assignment to node $h$. Otherwise, the number of inactive nodes other than node $i$ between nodes $h$ and $j$, $(\mathrm{j}-\mathrm{h}-2)$, and the number of inactive nodes between nodes $\mathrm{h}$ and $\mathrm{i},(\mathrm{i}-\mathrm{h}-1)$ are used to determine $\mathrm{Tb}$ of the assignment to node $\mathrm{i}$. Note that $(\mathrm{j}-\mathrm{h}-2)$ is the number of nodes that will probably become active after node $i$. $\mathbf{S}$, the space between assignments for nodes $h$ and $\mathrm{i}$, is reserved for the inactive nodes between nodes $\mathrm{h}$ and $\mathrm{i}$. When they become active, they can use $\mathbf{S}$ without shifting the positions of other assignments. The qEA implementation can be summarized as follows:

1) Find the active nodes closest to node $i$ on both sides of it, i.e., nodes $h$ and $j$;

2) Calculate the available bandwidth (B) between the assignments for nodes $\mathrm{h}$ and $\mathrm{j}$ according to the formula: $\mathrm{B}=\mathrm{Tb}(\mathrm{j})-\mathrm{Te}(\mathrm{h})+d(1, \mathrm{j})-d(1, \mathrm{~h})$;

3 ) If $B=0$, then skip to the next node to be honored and go to step 1 . The allocation process stops when the same node is encountered for a second time;

4) Calculate $\mathrm{q}, \mathrm{Tb}$ and Te according to the formula given above;

5) Same as step 4 in the SA implementation.

\subsubsection{Parameters Setting in $\mathrm{qEA}$}

The value of $\mathrm{M}$ influences the performance of the qEA scheme. If $\mathrm{M}$ is set to L2 / $n$ and each node has enough traffic (i.e., $\mathrm{R} \geq \mathrm{M}$ ), the whole cycle is shared by all the nodes in a fair manner. In this case, there is no wastage in the cycle as shown in Figure 4. This setting is called average setting. Notice that a cycle with this setting does not imply the bandwidth utilization is always 1 due to the reason illustrated in Figure 5 (refer to Section 2.2.a for details). However, with this setting, the bandwidth wastage is small since the assignment to a node per cycle is relatively small and the traffic from each node can often be satisfied with this assignment. If $\mathrm{M}$ is set smaller than L2 / $n$, the cycle will always have unused segments. The total unfilled bandwidth within a cycle can be calculated by $n *$ (L2 / $n-\mathrm{M})$ if all the nodes are active. The smaller $\mathrm{M}$ is, the larger the bandwidth wastage. There is also bandwidth wastage if $\mathrm{M}$ is set much larger than $L 2 / n$. The following is the analysis for the case $\mathrm{M}=\mathrm{L} 2-\mathrm{I}$, which will be called maximum setting. 


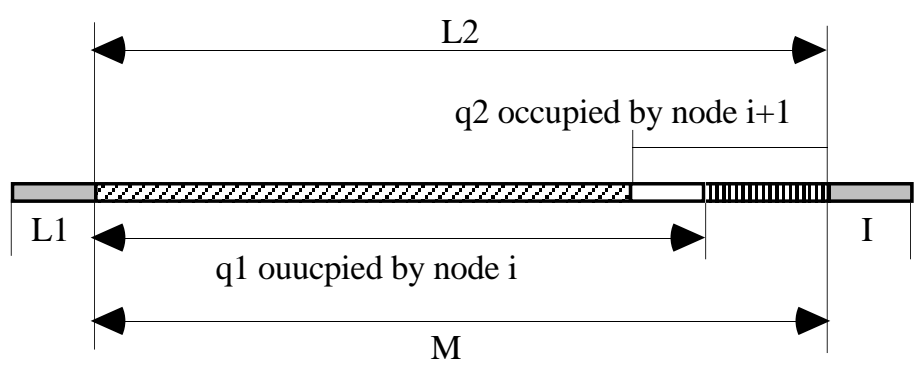

$\square$ is the propagation delay between nodes $\mathrm{i}$ and $\mathrm{i}+1$

Figure 11 Cycle division with maximum M setting

With the maximum setting, if traffic from each node can always use up the quota (i.e., M) in each cycle, the bandwidth wastage per cycle is relatively small and is equal to I as shown in Figure 11. If the traffic from each node (e.g., node i in Figure 11) cannot use up the quota but takes the majority of $\mathrm{M}$ (i.e., q1 in Figure 11), a small segment q2 is left to node $i+1$. It is clear that node $i$ can quickly complete its transmission with a large quota q1 and immediately release its assignment upon completion. According to the principle of the EA scheme, the assignment released by node $\mathrm{i}$ can only be used by node $\mathrm{i}$ or its downstream nodes such as node $\mathrm{i}-1$ if node $\mathrm{i}+1$ has not released its assignment (see Figure 5). If neither node $i$ nor its downstream nodes are active, the released assignment is wasted. Moreover, it will take a long time for node $i+1$ to finish its transmission with the small quota $\mathrm{q} 2$. In the worst case when $\mathrm{i}=1$, the bandwidth wastage is very large. Therefore, a reasonable $\mathrm{M}$ setting is the average setting.

\section{Simulation Studies}

In this section, we present simulation results for the prototype presented in Section 3. As mentioned in Section 2.1, there are two types of requests that a node can make, i.e., GBR and CBR. With CBR, a node can get the guaranteed bandwidth in every cycle if its CBR request is confirmed. This is similar to GBR when a large GBR request is divided into multiple fragments served by several consecutive cycles. Therefore, our simulation is concerned with GBR requests only.

\subsection{Simulation Model}

The simulation model consists of 25 nodes with equal distance between any two neighboring nodes. The network size (NS) is equal to $24 * \mathrm{~s}$ (assuming the distance 
between node 1 and the headend is 0 ). The traffic model of each node is a bursty pulse train [10] as shown in Figure 12. The delay between two consecutive bursts and the number of cells in a burst follow respectively the exponential and geometric distributions. The network capacity is set to $155.52 \mathrm{Mbps}$ corresponding to OC-3, which results in the cell length (CL: time to transmit an ATM cell) of $2.726 \mu \mathrm{s}$. The static reservation for each node is set to $\mathrm{s}$. The length of the static reservation segment in a cycle, L1, is equal to $25 * \mathrm{~s}$. The mean cell arrival rate from a node (MCR) can be computed by BL / (DB + BL * IC). The cell transmission rate given by the network is equal to 1 / CL, which is shared by 25 nodes so that each node gets $1 /(25 * \mathrm{CL})$. The offered load (OL) is defined as MCR / [1 / $(25 * \mathrm{CL})]=25 * \mathrm{CL} / \mathrm{MCR}$. Given CL, OL and BL, DB $=(25 / \mathrm{OL}-1) * \mathrm{BL} * \mathrm{CL}$.

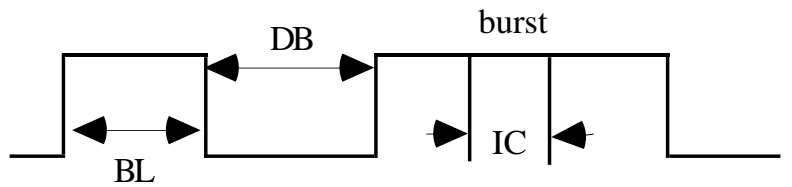

\footnotetext{
DB: mean delay between two burrsts (exponential distribution)

BL: mean number of cells in a burst (geometric distribution with probability $=1.0 /[\mathrm{BL}+1])$

IC: interval between two cells (constant equal to a cell length)
}

Figure 12 Burst pulse train traffic model

The main performance index to be investigated is bandwidth utilization $\mu$. The request response time is not measured since a node can continue to make requests even after the previous ones have not been honored; and the headend may honor several requests for a given node at the same time. Table 1 lists the main parameter values used by the different simulations $(\mathrm{NS}=$ network size, $\mathrm{BS}=$ buffer size, $l=$ cycle length, $\mathrm{OL}=$ offered load, $\mathrm{BL}=$ burst length, $\mathrm{PV}=$ parameter to be varied). The default $\mathrm{M}$ setting is (L2 - I) for the SA scheme with $\mathrm{I}=d$, and L2 / 25 for the qEA scheme. 


\begin{tabular}{|c||c|c|c|c|c|}
\hline Figure & $\begin{array}{c}\text { N S } \\
(\mathbf{k m})\end{array}$ & $\begin{array}{c}\text { B S } \\
(\text { cell) }\end{array}$ & $\begin{array}{c}\boldsymbol{l} \\
(\text { cell) }\end{array}$ & OL & $\begin{array}{c}\text { BL } \\
(\text { cell })\end{array}$ \\
\hline \hline $\mathbf{1 3}$ & 13 & 2500 & PV & 1.0 & 30 \\
\hline $\mathbf{1 4}$ & 13 & 2500 & PV & PV & 30 \\
\hline $\mathbf{1 5}$ & 13 & PV & 300 & 0.75 & PV \\
\hline $\mathbf{1 6}$ & 13 & PV & 300 & 0.75 & PV \\
\hline $\mathbf{1 7}$ & 13 & PV & 300 & 0.75 & PV \\
\hline $\mathbf{1 8}$ & PV & 2500 & PV & 1.0 & 30 \\
\hline $\mathbf{1 9}$ & PV & 2500 & PV & 1.0 & PV \\
\hline
\end{tabular}

Table 1 Parameter setting for the different simulations

\subsection{Simulation Results}

This subsection is organized as follows: Figures 13 to 17 investigate the effects of some parameter settings (e.g., request threshold, offered load and buffer size) to the performance of SA and qEA schemes; and Figures 18 and 19 compare the performance between SA and qEA. Although qEA shows better performance than SA in many cases as shown below, the main advantages of SA are its simplicity and applicability to wireless networks. The simulation results are obtained using the simulation package BONes DESIGNER V 2.6 from Alta Group ${ }^{\mathrm{TM}}$. Each point on the graphs took about 5 hours to simulate on a SPARC5 machine.

Figure 13 shows the effect of request threshold (T) to bandwidth utilization $(\mu)$ for SA (in left figure) and qEA (right figure). It is observed that T has very little effect on $\mu$. For SA, as mentioned in Section 3.2.1, a reasonable cycle setting (e.g., CL = 300) can produce a good $\mu$. For qEA, the cycle length is 500 cells and $M$ is set to $15,19,25$ (average setting) and 451 (maximum setting) cells respectively. The results confirm the assertion mentioned in Section 3.2.2 that the average setting gives better $\mu$. Note that SA can almost achieve the same $\mu$ as qEA, but the number of simultaneous active nodes in SA is limited to 1 or 2 by the maximum $\mathrm{M}$ setting (i.e., $\mathrm{M}=\mathrm{L} 2$ - I) while that in qEA is equal to $\mathrm{N}$ (e.g., $\mathrm{N}=25$ in this simulation model). (Refer to Figure 6 in Section 2.2 which depicts $\mu$ versus the number of simultaneous active nodes.) 


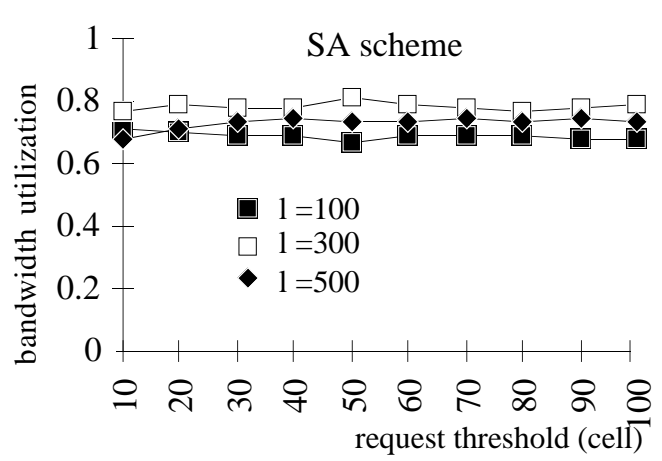

a) $\mu$ vs $1, M=1-50$ cells

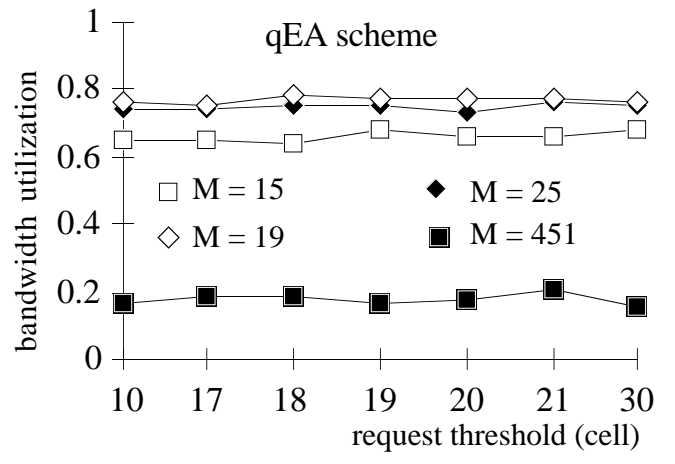

b) $\mu$ vs $\mathrm{M}, 1=500$ cells

Figure 13 Effect of request threshold to bandwidth utilization

Figure 14 depicts bandwidth utilization $(\mu)$ versus offered load (OL) for different cycle lengths $(l) . \mu$ at T is the theoretical maximum value (TM $\mu)$ for a given network size and cycle length according to the analyses in Section 2.2. Note that it is easier for traffic to fill a shorter cycle (e.g., $l=100)$ rather than a longer one. However, increasing $\mu$ with increasing OL in SA with a short cycle is limited by the TM $\mu$ which is smaller than 1 in SA. It is possible to have a high $\mu$ in qEA since the TM $\mu$ is equal to 1 . In addition, different cycles in qEA lead to the same $\mu$ in the case of heavy load since $\mu$ given by qEA is not influenced by the cycle setting but mainly depends on the traffic pattern of the nodes like the EA scheme mentioned in Section 2.2.
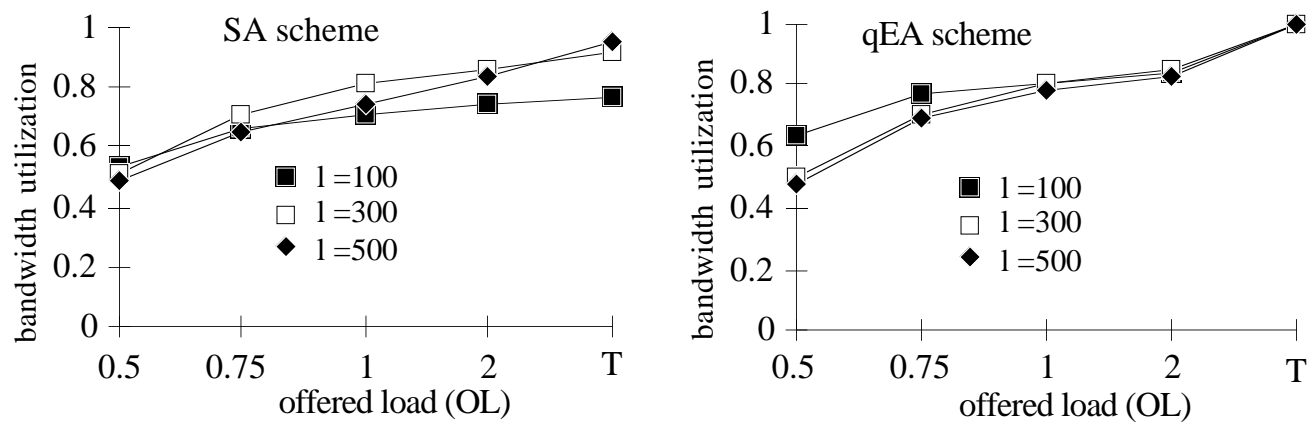

Figure 14 Bandwidth utilization versus offered load

Figures $15 \sim 17$ show the performance (bandwidth utilization, mean response time and mean cell loss rate) for different buffer sizes (BS) and different burst lengths (BL). Generally, when the buffer is large enough, the influence of BL becomes weak in SA, but this is not the case in qEA. It is because $\mathrm{M}$ is set to the maximum in SA so that a node can 
get a larger quota in each cycle. After finishing the quota, the node needs to wait for a long time to get another quota. During this period, the large buffer can be used to smooth the traffic. However, qEA adopts the average setting so that a node can usually get a quota in each cycle. This makes qEA sensitive to traffic burstiness. Therefore, qEA with the average setting is more suitable for less bursty traffic.
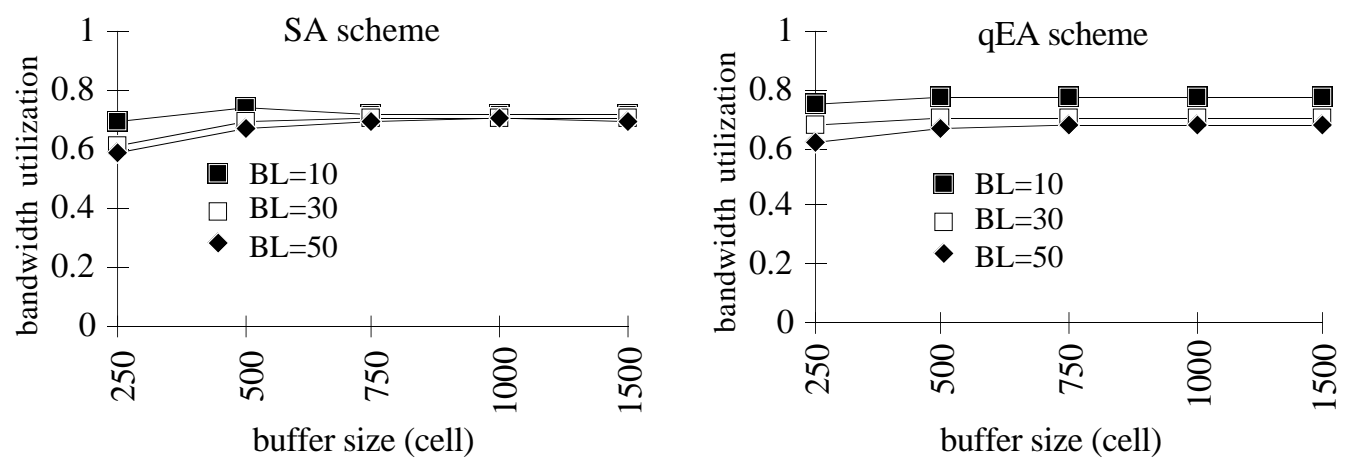

Figure 15 Bandwidth utilization versus buffer size

Figure 16 shows the mean response time (MRT) as a function of buffer size. MRT is the elapsed time between the cell arrival to the buffer and its departure. Different from $\mathrm{SA}, \mathrm{MTR}$ in qEA is sensitive to BL due to the reason mentioned above.
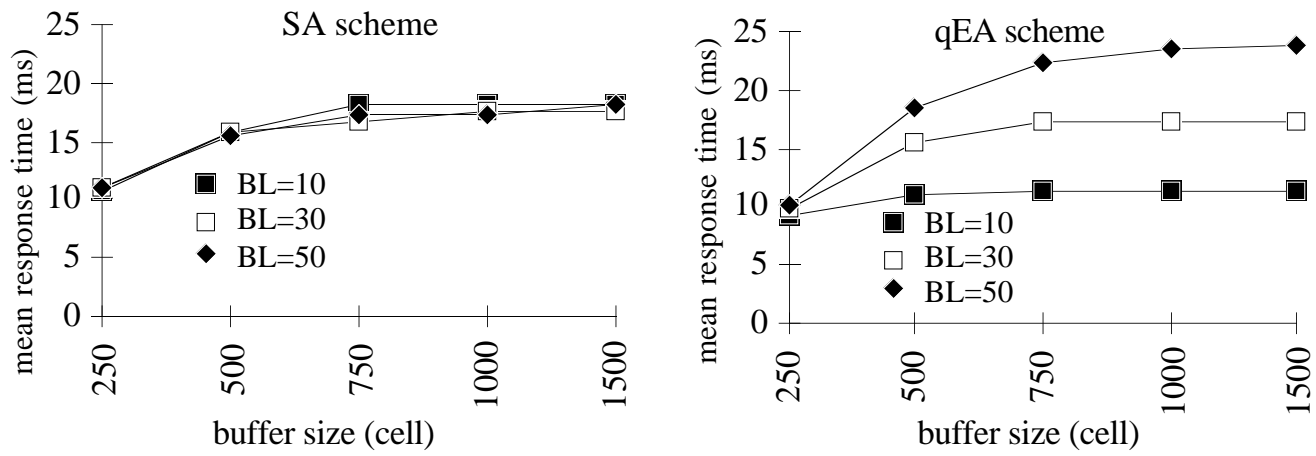

Figure 16 Mean response time versus buffer size

Figure 17 presents the mean cell loss rate (MCLR) calculated in terms of total lost cells over total generated cells. When the buffer size is small, MCLR for qEA is smaller than that for SA because the bandwidth allocation in the cycle in qEA with the average setting is more suitable than that in SA with the maximum setting for the traffic accumulated 
in a small buffer. The effect is reversed in the case of large buffers due to the reason mentioned above.
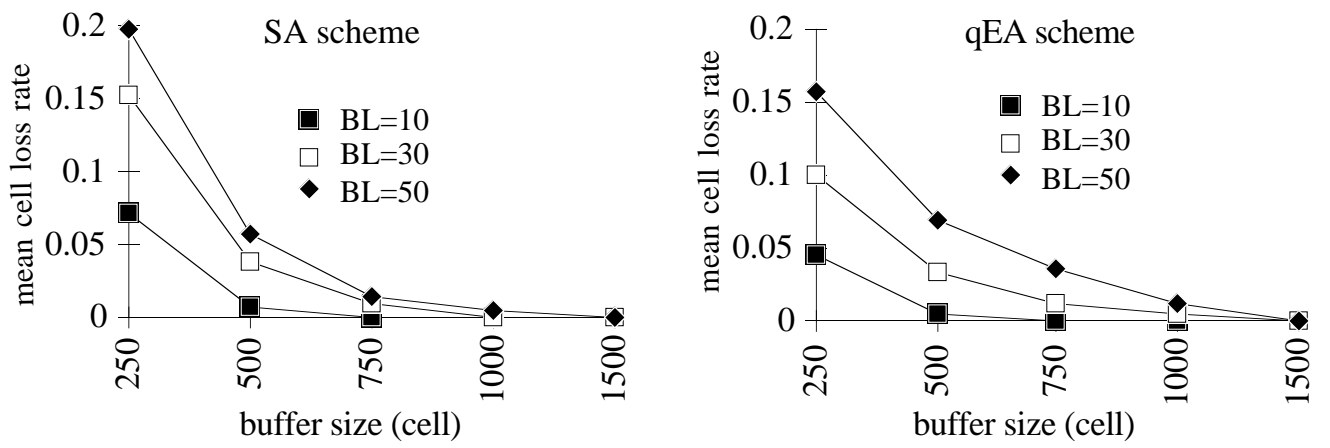

Figure 17 Mean cell loss rate versus buffer size

Figure 18 shows bandwidth utilization $(\mu)$ versus network size (NS) with cycle lengths of 300 and 500 cells. SA_t and EA_t are respectively the theoretical maximum $\mu$ for SA and EA respectively (refer to Section 2.2 for the calculation). It is clear that $\mu$ in SA decreases with increasing NS in the case of $l=300$. In the case of $l=500, \mu$ first increases with NS up to NS $=52$ then decreases. This is due to the mismatch between the assignment in the cycles and the traffic load from the nodes. Since long distance increases the response delay because of the propagation delay, this gives some time for the nodes to accumulate traffic. Similar observation is also found in qEA. However, this increase is limited by the theoretical maximum value. Anyway, qEA is less influenced by NS than SA so qEA is more suitable for metropolitan area of networks.
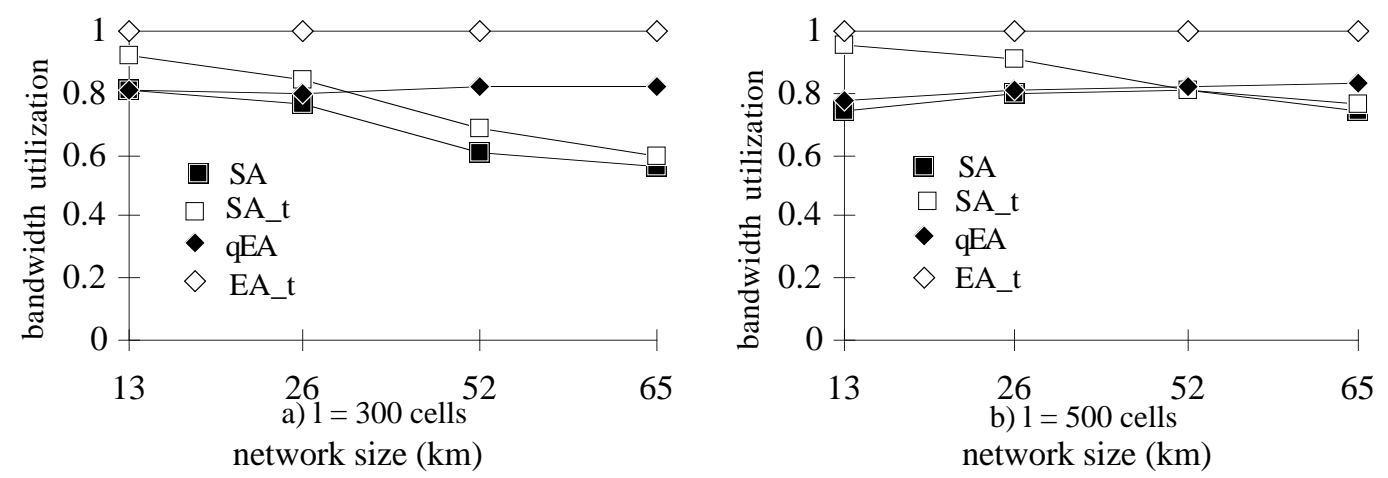

Figure 18 Bandwidth utilization versus network size 
The fairness with which the nodes share the upstream bandwidth depends on the service discipline used by the scheduling algorithm at the headend. In the simulation, the nodes are served one by one in round-robin rather than FIFO discipline since the latter will lead to unfairness caused by the propagation delay between the nodes and the headend. Figure 19 plots the mean access interval for each node, which is the mean interval between two consecutive accesses to the media for a node. As shown in Figure 19.a, qEA behaves better than SA since qEA use the average setting and SA uses the maximum setting (refer to Section 3.2.3 for details). As illustrated in Figure 19.b, SA performs very well in the case of burst length equal to 1 . A better service discipline is needed to ensure fairness among all nodes for $\mathrm{BL}>1$.

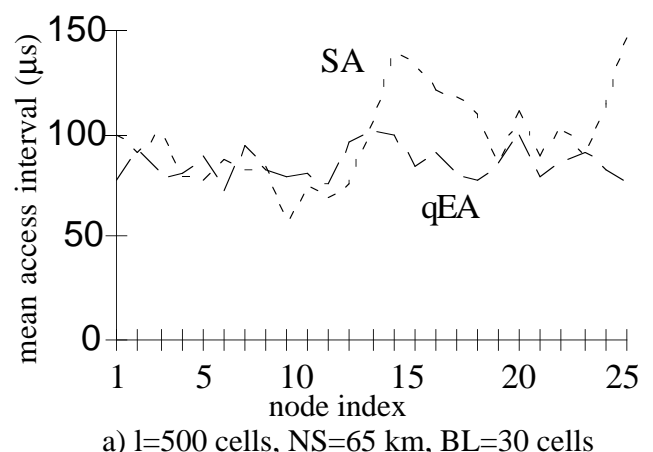

a) $l=500$ cells, $\mathrm{NS}=65 \mathrm{~km}, \mathrm{BL}=30$ cells

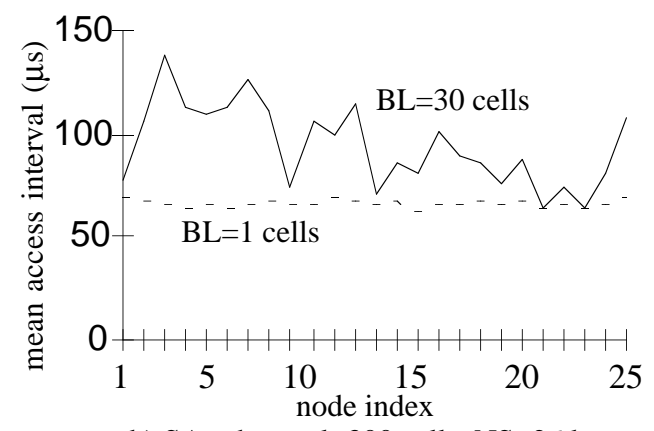

b) $\mathrm{SA}$ scheme, $\mathrm{l}=300$ cells, $\mathrm{NS}=26 \mathrm{~km}$

Figure 19 Fairness issue on mean access interval in SA and qEA

\section{Discussion}

A MAC scheme based on time division for the HFC system, SCCA/TD, was discussed in this paper. The reservation scheme with self-control-cyclic-access mechanism, which can be easily implemented with timers, allows SCCA/TD to provide the bounded delay and throughput. This scheme requires less control from the network and permits the nodes to be passively linked to the buses which enhance network robustness. The non-slotted media based framework can accommodate different transmission rates and support different types of MAC protocol data units. Although the priority mechanism has not been addressed, it can be easily implemented since the allocation policy is centrally performed by the headend. The static reservation segment (SRS) in each cycle can provide quick access to the medium. However, it causes bandwidth wastage when a node has nothing to transmit. The wastage can be alleviated by allowing several nodes to share the same SRS assignment alternately. The scheduling algorithm is the key in SCCA/TD. Two schemes were discussed in the paper: the simple one (SA) is suitable for small size networks and the efficient one (EA) 
suitable for a wide range of network sizes. More efficient schemes to realize EA require further studies.

Another open issue is to find the optimal setting for some parameters such as the cycle length $(l)$, the request threshold (T) and maximum quota (M). This can be done by theoretical analyses. The difficulty is to find a traffic model that matches well with real traffic. Another way based on measurements is for the headend to monitor the performance oscillation and adjust the parameter settings accordingly. This is feasible for the SCCA/TD framework since its parameters can be dynamically set. Moreover, with the capability of rearranging the positions of existing assignments in the cycles, the bandwidth allocation can be dynamically adjusted according to the traffic load on the network at the expense of complexity. In addition, SCCA/TD can be further developed to a hierarchical cycle scheme to satisfy different delay requirements of different applications, and can also be extended to wireless networks.

\section{References}

[1] J. W. Eng, J. F. Mollenauer, "IEEE Project 802.14: Standards for Digital Convergence", IEEE Communications Magazine, May 1995 Vol. 33 No. 5, pp20-23

[2] Mario P. Vecchi, "Broadband Networks and Services: Architecture and Control", IEEE Communications Magazine, August 1995 Vol.33 NO.8, pp24-32

[3] Kazuo Imai,Tadashi Ito,Hideki Kasahara et al., "ATMR: Asynchronous transfer mode ring protocol", Computer Networks and ISDN Systems 26 (1994) 785-798

[4] J.L. Adams, "Orwell", Computer Networks and ISDN Systems 26 (1994) 771-784

[5] James Martin, "Local Area Networks, Architectures and Implementations", 2nd Edition, PTR PRENTICE HALL, 1994

[6] E.A. Zurfluh, R.D. Cideciyan et al, "The IBM Zurich Research Laboratory's 1.13 Gb/s LAN / MAN Prototype", Computer Networks and ISDN Systems 26 (1993) 163-183

[7] Bandula W.Abeysundara, "High-speed local area network and their performance:a survey", ACM computing surveys,Vol.23,No.2,June 1991,p221-65

[8] D.J. Goodman, R.A. Valenzuela et al, " Packet Reservation Multiple Access for Local Wireless Communications", IEEE Transcations on Communications, Vol. 37, No. 8, 8 August 1989, p885-890

[9] Mischa Schwartz, "Telecommunication Networks, Protocols, Modeling and Analysis", Adison-Wesley publishing Company, 1988 
[10] Alta Group of Cadence Design Systmes (Inc), BONeS DESIGNER Core Library Reference, V 3.0 